\title{
Guest editorial introduction to the Special Issue on bio-hybrid systems and living machines
}

\author{
Leonardo Ricotti • Arianna Menciassi - Keisuke Morishima
}

Published online: 18 November 2012

(C) Springer Science+Business Media New York 2012

\section{Introduction}

Current research in robotics and mechatronics is more and more oriented towards the development of flexible, soft and high performance components able to mimic biological structures. The challenge of producing a novel generation of machines, able to overcome current technological limitations (mainly due to actuator, sensor and battery performances) will necessarily entail the communion of biology and micro/nano-technology.

Bio-hybrid systems foster the opportunity of integrating a properly engineered artificial structure and a living bio-system, to boost or to exploit the unique characteristics of biological systems or components, refined over millions of years of natural evolution, in order to support specific applications and to solve advanced or critical technological problems.

Living machines represent emerging and promising platforms for a wide range of applications, ranging from the microscopic to the macroscopic domain, and related to the medical field, but also to many other exquisite technological issues.

\section{Recent works on bio-hybrid systems}

Bio-hybrid systems are emerging in the last years as a promising paradigm for engineering, aiming at solving technological issues by means of the integration of living biological

\author{
L. Ricotti $(\bowtie) \cdot$ A. Menciassi \\ The BioRobotics Institute, Scuola Superiore Sant'Anna, \\ Pontedera, Pisa, Italy \\ e-mail: 1.ricotti@sssup.it \\ A. Menciassi \\ e-mail: a.menciassi@sssup.it \\ K. Morishima \\ Department of Mechanical Engineering, Osaka University, \\ Osaka, Japan \\ e-mail: morishima@mech.eng.osaka-u.ac.jp
}

components, which are already optimized by nature. This would allow the exploitation of the unique features of living cells and tissues not for mimicking them, but for physically integrating them in a properly engineered and controllable artificial structure. Conversely, the use of bio-hybrid systems raises a series of side technical problems (mainly related to the issue of a long-term maintenance of the living tissue) that must be addressed to assure system functionality, efficiency and reliability.

A wide picture can be drawn in the framework of biohybrid systems and living machines, aiming at tracing a parallel between the traditional mechatronic scheme, constituted by the well-known blocks of sensors, actuators, mechanisms, control systems and energy supply, and the novel technologies that are arising from the symbiosis between artificial and living entities.

Functional microdevices fitting in the human eye and taking over lost biological functions to restore vision have been recently proposed as bio-hybrid retinal implants. Although a series of technical and biological problems remain still unsolved at present, these studies demonstrated that a retina prosthesis based on engineered materials and living cells is technically feasible and could be strongly fostered in the near future thanks to the expected advances in microand nano-technologies. In parallel, a wide range of bacteriabased sensors were developed in the last years, mainly to accomplish chemical sensing functions.

Robot control issues were also recently addressed through a bio-hybrid approach, by exploiting the advanced strategies developed by living systems to overcome computationally difficult challenges. A plasmodium was recently integrated into a robot controller to import the plasticity and adaptability of a living organism into a device architecture, thus demonstrating the possibility to obtain integrated biohybrid information processors, at least for niche applications. Cell-based controllers are actually expected to show better performances in comparison with traditional ones, 
especially when facing contradictory or undefined situations. Rat and human neurons have been also cultured on artificial chips, with the aim of creating bio-hybrid neural networks able to drive robot locomotion or to accomplish other functions by exploiting the learning ability of living neurons.

The molecular recognition properties of DNA molecules, combined with the mechanical properties of single and double strands of DNA can be utilized for the construction of nanodevices. These systems can be considered nanoscale mechanisms allowing rotational motion, pulling, stretching, etc. Specific mechanisms such as miniaturized gears or tweezers have been recently developed using such molecules.

Concerning bio-hybrid energy supply systems, the possibility of exploiting the autonomous beating ability of rat cardiomyocytes to efficiently convert chemical energy (deriving from glucose) into mechanical energy was recently proposed. Cells were cultured on artificial substrates and interfaced with a PZT fiber that generated voltage by piezoelectric effect. A musclebased system to power microelectronic devices has been also proposed.

Living muscles have evolved over millions of years within animals as nature's premier living generators of force, work and power, showing unique features in comparison with standard artificial actuators. Within its motor function, muscle acts providing positive mechanical work at a considerable aerobic transduction efficiency, with $1,000 \mathrm{~J}$ of work per gram of glucose consumed. Actuation systems relying on muscle tissue engineering, based on contractile cell organization onto engineered substrates, is recently emerging and it would open new horizons for engineering and robotics. This motivation paved the way to a recent series of studies, in which self-beating or skeletal muscle cells were coupled with artificial micro-systems, thus opening the frontier of the so-called "wet robotics".

\section{Recent highlights of this Special Issue}

The present Special Issue is composed of 14 articles, grouped in four main categories, namely:

a) Bio-hybrid actuators.

b) Bio-hybrid sensors.

c) Bio-hybrid power sources.

d) Enabling technologies for bio-hybrid systems.

The first category is the most represented one, with eight articles. Three of them concern actuation mediated by living mammalian or insect cells, while the other five describe devices and systems moved by bacterial cells.

Hoshino et al. describe an engineered gel moved by cardiomyocytes cultured within its structure. The gel represents a network that permits electrical potentials and mechanical stretch-induced contractions, thus providing not only a wet robotic system but also a platform to investigate how cardiac diseases related to electrical propagation occur.

Akiyama et al. report an insect muscle-powered autonomous robot, constituted by an artificial flexible frame and dorsal vessel contractile insect tissue. The robot moves autonomously by spontaneous contraction of the living tissue and its velocity is increased by means of chemical stimulation.

Ricotti and Menciassi report a detailed review of the state of the art concerning muscle cell-based actuators. The main limitations of current actuation technologies and the envisioned benefits that a shift towards the bio-hybrid paradigm could imply are reported, together with a description of the most recent scientific and technological efforts to achieve efficient cell- or tissue-based actuators.

Sahari et al. investigate how micro-robot shape can influence the motile behavior of bacteria-powered swimming devices. The authors describe how viscous forces, developed at the interface of the swimming micro-robots and the surrounding fluid, change with body geometry.

Kim et al. highlight the possibility of combining the motility of flagellated bacteria ( $S$. Marcescens) with engineered polystyrene micro-beads, thus creating a bio-hybrid system able to propel itself towards an attractive chemical source.

Cho et al. propose a fabrication method of biocompatible poly(ethylene glycol) micro-beads selectively functionalized with poly-L-lysine. The authors report that bacterial cells adhere only on the functionalized regions of the micro-beads, thus enhancing device velocity and allowing directionality.

Kojima et al. combine bacteria and liposomes by means of biotin-streptavidin binding technique to create bacteriadriven liposomes. A novel binding technique is proposed, to limit the attachment of bacteria to small areas of the liposome, thus leading to a more efficient motility of the system.

Finally, Martel reports a detailed review of the state of the art concerning motile bacteria-based bio-hybrid devices. Many techniques and approaches are also described, which can be considered as building blocks for the implementation of more sophisticated micro-robots and micro-systems.

The category "Bio-hybrid sensors" includes two articles, both aimed at describing devices constituted by biological sensing components.

$\mathrm{Wu}$ et al. describe an aptamer-based biosensor for the detection of ATP locally secreted from single taste receptor cells. DNA aptamers are used as recognition elements and their DNA competitors serve as transduction elements.

Liu et al. report a bio-hybrid olfactory system constituted by living rat olfactory cells interfaced with micro-electrode arrays, able to record the odor-induced electrophysiological activity of the tissue. 
Shoji et al. report the development of a biofuel cell based on insect hemolymph, thus contributing with an interest approach to the field of "Bio-hybrid power sources". The maximum power density demonstrated by Shoji using the insect-based biofuel cell is $6.07 \mu \mathrm{W} / \mathrm{cm}^{2}$.

The category "Enabling technologies for bio-hybrid systems" includes three articles, facing different technological issues connected with the development and maintenance of artificial structures coupled with living entities.

Fujie et al. describe an interesting method to provide poly (methylmethacrylate) nanofilms with a surface protein patterning, thus achieving cell alignment on such substrate for new generations of muscle-based actuators.

Islam et al. demonstrate the alignment of polystyrene latex micro-spheres, functionalized superparamagnetic beads and live cells using an ultrasonic standing wave in a PDMS microfluidic channel. This technology could allow new functionalities for bio-hybrid sensor systems and bio-assays.

Finally, Masuda et al. describe a technique to assemble cells in three-dimensional spheroid micro-tissues. These structures could allow in the near future the development of novel bio-hybrid actuators or parts of micro-machines.

\section{Future trends of bio-hybrid systems}

The findings described in this Special Issue suggest that biohybrid solutions represent a promising paradigm for future technological issues, with a disruptive potential especially concerning the fields of soft and adaptive robotics.

To permit the bio-hybrid paradigm to express all its potential, new enabling technologies will be needed, in order to properly address the numerous issues connected to the use of living cells within artificial devices (e.g. maintenance of cell viability and functionality, force/signal transmission, etc.).

We expect that a new generation of scientists could be fostered by the bio-hybrid approach, whose future achievements will necessarily require an unprecedented interdisciplinary, yet integrated effort.

Acknowledgment As Guest Editors, we thank all the contributing authors for the submission of their latest research results on bio-hybrid devices and enabling technologies to this Special Issue. We are also thankful for all the reviewers and their hard work, which allowed this Special Issue to be published on the planned schedule. Finally, we thank Prof. Mauro Ferrari for giving us the exciting opportunity of organizing this Special Issue. 